\title{
Jóvenes, música y pantallas. Evolución formal y de contenido de los 40.com y adaptación a los dispositivos móviles
}

\section{Youth, music and displays. The case of los40.com. Evolution of form and content of the site and mobile applications.}

\author{
Dra. Ana Isabel Bernal Triviño \\ Investigadora grupo Labcom \\ Universidad de Málaga (UMA) \\ anaisbernal@uma.es
}

Recibido: 06 de noviembre 2012 Aceptado: 27 de noviembre de 2012

\section{Resumen}

Los jóvenes dedican gran parte de su tiempo a las nuevas tecnologías y a escuchar música. En España, el medio líder de información musical en Internet es los40.com. Este artículo realiza un análisis exploratorio formal y de contenido del portal y de su adaptación a las aplicaciones móviles de los sistemas operativos Android, Symbian, e iOS (iPhone- iPad), para describir su evolución desde el año 2008 hasta 2012. La justificación de esta investigación reside, en primer lugar, para completar la deficiencia de estudios sobre el diseño periodístico de los contenidos en los cibermedios musicales y en la tecnología móvil y; en segundo lugar, para evaluar si existe una adaptación de los40.com a las preferencias de los jóvenes, en un contexto de convergencia tecnológica. El análisis se concentra en la estructura, tipografía, multimedia, contenidos, documentación/actualización y participación. Los resultados muestran una adaptación paulatina de contenidos y de rasgos formales, adaptándose e integrando nuevas secciones en función del desarrollo de la industria de contenidos digitales. En los dispositivos móviles, se detecta una distribución multiplataforma con adaptación formal, pero no de contenido. Sí destacan, en todas estas aplicaciones, su valor de movilidad a través de la escucha online desde los dispositivos y su potenciación de las redes sociales y el multimedia. 


\begin{abstract}
Young people spend much of their time to the new technologies and music. In Spain, the leading medium of music information on the Internet is los40.com. This article takes a formal exploratory analysis of portal content and applications to adapt to mobile operating systems Android, Symbian, and iOS (iPhone, iPad), to describe its evolution from 2008 to 2011. The justification of this research is, first, to complete deficiency newspaper design studies of the music contained in the online media and mobile technology and, second, to assess whether there is an adaptation to los40.com preferences of young people, in a context of technological convergence. The analysis focuses on the structure, typography, multimedia, content, documentation / updating and participation. The results show a gradual adaptation of content and formal features, adapting and integrating new sections based on the development of the digital content industry. On mobile devices, multiplatform distribution is detected with in form, but not content. Yes highlighted, in all these applications, the value of mobility through listening online from devices and empowerment of social networking and multimedia.
\end{abstract}

Palabras Clave: periodismo; internet; imagen; multimedia; hipertexto; información.

Key Words: journalism; internet; image; multimedia; hypertext; information.

\title{
Sumario
}

1. Introducción: jóvenes, música y pantallas. 2. Metodología. 3. Resultados. 4. Discusión. 5. Conclusiones. 6. Bibliografía.

\section{Summary}

1. Introduction: youth, music and displays. 2. Methodology. 3. Results. 4. Discussion. 5. Conclusions. 6. Bibliography.

\section{Introducción: jóvenes, música y pantallas}

Más del 90\% de los jóvenes dedica parte de su tiempo a oír música (Funes, 2008: 112). Desde una perspectiva histórica, las raíces de este consumo surgen en la sociedad norteamericana a partir de los años 50 (Hormigos y Martín, 2004: 262), arraigándose en el capitalismo durante los 70 e instaurándose el consumo de masas (Megías y Rodríguez, 
2003: 251). Es entonces cuando se integra en la industria cultural, concretada por Adorno (1973), donde los medios se desvelan como transmisores de estereotipos, produciendo una homogeneización en la cultura musical.

La música no es sólo un bien relacionado con el ocio, sino que aporta valores y emociones, y permite a las nuevas generaciones crear una "identidad colectiva" (Funes, 2008: 116). Es un instrumento con el que los jóvenes pueden divertirse, relacionarse con otras personas, y favorecer la integración, a pesar de poseer también un matiz diferenciador de grupos, como los clubs de fans (Megías y Rodríguez, 2003: 249). La radio musical, en ese sentido, reafirma ese sentimiento de grupo y de afirmación de los gustos musicales (García, 2010). En consecuencia, se puede concretar que la música desempeña en los jóvenes cuatro valores: identidad, ocio, relación y emotividad.

No obstante, no puede eludirse su valor económico, como propia industria (Sedeño, 2005: 2). Los jóvenes acceden a ella por diversos canales, como que los amigos se presten grabaciones, "bajar" gratis música de Internet o la compra en el mercado ilegal (Megías y Rodríguez, 2003: 252), área donde se trabaja con fuerza para concienciar a la población de este delito. Además se reclama a las discográficas nuevos modelos de negocio que aprovechen la red como un "cauce de distribución para la música online, a precios más competitivos" (Sedeño, 2005: 5). De ahí que hayan arraigado con tanto éxito, debido a las mejoras de conectividad, los servicios de streaming, como Spotify, al que accede un 64,9\% de la población española (Fundación Telefónica, 2012:54). No obstante, estas tendencias de consumo no se deben solo a las tecnologías, sino a las políticas públicas desarrolladas (Calvi: 2006, 133).

En la última década, las novedades tecnológicas han provocado notables modificaciones en los tiempos de ocio y de relación de los jóvenes. Se ha originado un proceso de "pantallización" de la sociedad (Rodríguez de las Heras, 1991: 49) y de digitalización, que ha llevado a que la juventud consuma un $90 \%$ de estos contenidos, como ver vídeos o escuchar música en Internet (Fundación Telefónica, 2011:15). Los jóvenes han desempeñado una función crucial en la aceptación de las nuevas tecnologías. De hecho, los hogares con niños presentan una penetración de servicios tecnológicos superior a la media de la población (Gimeno, 2011: 168). Los jóvenes conforman una generación "autónoma y autodidacta", que aprovecha al máximo las pantallas para relacionarse con los demás (Bringué y Sádaba: 2009, 12). Además, se caracterizan por un consumo simultáneo de medios (Fumero et alt., 2007: 53). Por ejemplo, el 53,4\% reconoce que navega mientras consume contenido multimedia (Fundación Telefónica, 2012: 26). Un hábito que también se centra, sobre todo, en el consumo de la radio y de Internet (Bernal, 2009 :130). 
El teléfono móvil, los videojuegos, e Internet son las tres tecnologías de principal uso por la juventud española. En cuanto al móvil, su posesión se localiza desde tramos de edad muy bajos. El 29\% de los niños a partir de 6 años reconocen tener uno, llegando a alcanzar el 94\% a los 16 años (Bringué y Sádaba, 2009: 21). En 2011 se ha producido un notable crecimiento de los smartphones, superando a la venta de PC (Fundación Telefónica, 2011:50). Este dato lleva a que el 76\% de los jóvenes universitarios consideren que los dispositivos móviles son los equipos tecnológicos más importantes de sus vidas (Fundación Telefónica, 2012: 51). El 64\% de los jóvenes españoles usan el smartphone para oír música o radio, y el 52\% para hacer fotos o grabar vídeo. Es decir, se imponen otros usos ajenos al de la comunicación con el móvil, como un soporte multiplataforma portátil (Bringué y Sádaba, 2009: 21). Hay determinados servicios que piden específicamente los jóvenes en sus smartphones como el GPS, la pantalla táctil, WIFI y el correo electrónico; junto a servicios como grabación de vídeo, envío de fotos, radio y MP3 (Gimeno, 2011:82). Es en este sentido, en el audiovisual, en el que Galindo (2008: 136) asegura que solo tendrán éxito aquellos que ofrezcan una "comunicación exclusiva" con el usuario.

La videoconsola es "un dispositivo asociado a jóvenes entre 15 y 19 años" (Funes, 2008: 160). El 90\% de los niños a partir de los 6 años reconoce jugar con videojuegos. A partir de ahí decrece su uso, hasta el 30\% a los 18 años (Bringué y Sádaba, 2009: 160).

El 95,3\% de los jóvenes de entre 16 y 24 años usa Internet (INE, 2011), dato que se reduce muy poco en edades inferiores, al 80\% de los niños de 10 años (Gimeno, 2011: 166). El $64 \%$ de los estudiantes reconocen que la red es "parte integral de sus vidas" y que es vital como "el aire, el agua, la comida o la vivienda" y el 65\% de las personas de entre 18 y 24 años elegirían Internet si tuvieran que quedarse con un solo medio durante todo un mes (Fundación Telefónica, 2011:54). El 52,4\% reconoce que han dejado de estudiar o leer para acceder a Internet; un 33,4\% ha disminuido el consumo de televisión; y un 23,6\% de los videojuegos (Bringué y Sádaba, 2009: 24-28). No obstante, estos cambios desafían las estructuras de negocio de las empresas periodísticas que deben enfocar nuevos planteamientos, dirigidos a la gratuidad de los contenidos y la adaptación de soportes más solicitados por la juventud. Son casos como el cierre de edición impresa de Super Pop (Elpais.com, 2011), la emisión de contenidos en abierto en el caso de MTV Spain (Elpais.com, 2010) o el acceso gratuito de la edición de Revista 40 en la red. De hecho, las nuevas tecnologías ya han provocado modificaciones considerables en la radio y sus sitios web, en relación a su programación (Murelaga, 2007). Los contenidos más visitados en Internet por los jóvenes son las web de música, los deportes y los juegos, la temática también más frecuente de sus propias páginas web y blogs (Bringué y Sádaba, 2009). A esta tendencia se añaden otras herramientas como los 
contenidos bajo demanda, visualizar vídeos en la red, descargas de música, chats y las aplicaciones de juegos de los smartphones (López, 2011:4).

Internet se ha convertido para los jóvenes en un espacio de reunión, apoyándose en las nuevas formas de interactividad, como las redes sociales (Onsti, 2011: 59). El 71\% de los adolescentes afirma utilizar las redes sociales. A partir de 14 años lo hace un $80 \%$, y a partir de los 17, un 85\% (Bringué y Sádaba, 2009: 18). El 91\% de los adolescentes utilizan Tuenti, y se trasladan a Facebook conforme se hacen adultos (Sánchez, 2011).

\section{Metodología}

\subsection{Objetivos}

Si Internet y el móvil son dos de las tecnologías preferidas por los jóvenes, y la música, uno de sus contenidos más solicitados, sería natural que la mayoría del consumo informativo o de ocio en la red se realice en esa línea. Entre los medios especializados, la radio musical está entre las preferidas por los españoles (AIMC, 2012). La integración adecuada de los contenidos de texto (noticias), audio (radio online) y vídeo (videoclip) en el cibermedio musical lo convierte en un factor básico para que los jóvenes acudan a él, siempre que éstas cumplan con sus expectativas.

El fin de este estudio es el de realizar un análisis evolutivo formal y de contenido de la web de los40.com y su adaptación a los nuevos soportes móviles. Se opta por este doble campo de estudio ya que su adaptación formal conlleva, a su vez, una adaptación de los contenidos. Los objetivos de esta investigación son los siguientes:

a)Describir la evolución formal y de contenido del site.

b)Describir la adaptación formal y de contenido del site a los nuevos dispositivos móviles, para detectar si se trata de un producto informativo nuevo o una copia de su referente.

c) Detectar si existe una vinculación entre los cambios producidos en la evolución formal y del contenido y los hábitos y preferencias descritos en el epígrafe 1.

\subsection{Metodologías previas}

Al margen de los informes estadísticos citados en el epígrafe anterior, que ayudan a dibujar un panorama sobre los hábitos y tendencias de los jóvenes en España, existe una deficiencia notable de estudios científicos sobre los cibermedios musicales. Sobre la relación de los jóvenes y los medios destacan los trabajos de Romero y Degrado (2005), Naval y Sádaba (2005), Pino y Galán (2010), Hormigos y Martín (2004), junto a otros dedicados a la 
música, las nuevas tecnologías y la radio (Burset y Sánchez, 2009; Requejo y Herrera, 2009; Turtós, 2008; Amoedo et alt. 2006). La mayoría de los estudios internacionales se centran en la música popular y cultural (Quirk y Toynbee, 2005; Atton, 2009). Las principales metodologías aplicadas son el enfoque exploratorio (Brushjensen y Larsen, 2010; Holt, 2011), o el análisis cualitativo de caso (McLeese, 2010; Preston y Rogers, 2011; Brennan, 2005; Jones, 2002; Forde, 2001).

En el diseño de la información en Internet se han registrado diversos enfoques, aunque no aplicados a los cibermedios musicales. Las primeras, de metodología descriptiva y análisis de contenido (Canga, 2005; Armentia, 2007; Cabrera, 2009; Nielsen, 2001). En el campo experimental están los estudios Eyetrack, del Poynter Institute. De él se extraen tres preferencias: los titulares grandes; el texto como punto de entrada a las páginas; y los párrafos cortos. También destacan algunos estudios más concretos, como la credibilidad en Internet (Yang y Patwardhan, 2004), o las preferencias visuales y textuales de los jóvenes en las noticias publicadas en los cibermedios (Bernal, 2009), con conclusiones como el predominio de los componentes audiovisuales para atraer a la audiencia juvenil, un mayor uso del color, titulares más grandes, párrafos cortos y separados, y tipografías sans serif. En cuanto a los nuevos dispositivos móviles destacan trabajos descriptivos (Meier, 2011; Lawson, 2010; o Goggin, 2010) e informes cuantitativos (Eye-Square, 2010; Change Wave Research, 2011; Nielsen Wire, 2010). El informe de Eye-Square (2010) concluye que las tabletas imponen que los contenidos periodísticos sean adaptados visualmente al nuevo dispositivo.

No se localizan investigaciones de cibermedios musicales centradas en el contenido, solo estudios globales (Riffe et alt., 2005), otros basados sobre los contenidos en Internet, en general; (Díaz Noci et alt. 2010:167; Navarro, 2011), así como de la web semántica (Sánchez, 2008).

\subsection{Método y material}

Debido a la escasa investigación focalizada en los cibermedios musicales se realiza una investigación descriptiva exploratoria, uno de los fines más comunes del estudio de caso (Yin, 1993:12); sin perseguir el interés de la generalización (Igartua, 2004: 93). Se usa una ficha de análisis de contenido como herramienta para identificar las características y propiedades del cibermedio analizado (Hernández et alt., 2006: 413). Se ha optado por un estudio de caso, de los40.com, enfoque "esencial" en las ciencias sociales (Yin, 1993: 11). Ante el panorama de crisis económica actual se hace necesario redefinir estrategias informativas y de negocio para aportar soluciones solventes en este sistema mediático, donde cada vez hay más soportes y una mayor diversificación de los ingresos. En 
consecuencia, solo analizando los casos de éxito y de pervivencia de los medios actuales se podrá aprender a definir tendencias de futuro (Bernal y Cabrera, 2011:2). Por lo tanto, se trata de un análisis específico, no representativo de los cibermedios musicales en general, sino que los resultados se encuentran condicionados por la muestra seleccionada. De hecho, autores como Krippendorff (2004:89) o Kohlbacher (2006:3) subrayan la compatibilidad del enfoque cuantitativo y cualitativo con los análisis de contenido.

\section{Tabla 1. Ficha de análisis}

\begin{tabular}{|c|c|c|c|c|}
\hline \multicolumn{5}{|l|}{$\begin{array}{l}\text { Variables } \\
\text { Medio, fecha y URL: }\end{array}$} \\
\hline \multicolumn{5}{|l|}{ Estructura } \\
\hline $\begin{array}{l}\text { Bloques: } \\
\text { Columnas: } \\
\text { Filas: } \\
\text { Espacio publicitario: }\end{array}$ & & $\begin{array}{l}\text { Ausencia: } \\
\text { Ausencia: } \\
\text { Ausencia: } \\
\text { Emplazamiento: }\end{array}$ & $\begin{array}{l}\text { Presencia: } \\
\text { Presencia: } \\
\text { Presencia: }\end{array}$ & $\begin{array}{l}\text { Emplazamiento: } \\
\text { Emplazamiento: } \\
\text { Emplazamiento: }\end{array}$ \\
\hline $\begin{array}{l}\text { Menús } \\
\text { Cabecera: } \\
\text { Color corporativo: } \\
\text { Blancos: } \\
\text { Localización temática en color: } \\
\text { Fondo: }\end{array}$ & & $\begin{array}{l}\text { Ausencia: } \\
\text { Ausencia: } \\
\text { Ausencia: } \\
\text { Ausencia: } \\
\text { Ausencia: } \\
\text { Ausencia: }\end{array}$ & $\begin{array}{l}\text { Presencia: } \\
\text { Presencia: } \\
\text { Presencia: } \\
\text { Presencia: } \\
\text { Presencia: } \\
\text { Presencia: }\end{array}$ & $\begin{array}{l}\text { Emplazamiento: } \\
\text { Emplazamiento: }\end{array}$ \\
\hline \multicolumn{5}{|l|}{ Tipografía } \\
\hline $\begin{array}{l}\text { Diferencia de cuerpo entre titulares } \\
\text { Familia (titular y entradilla) } \\
\text { Cuerpo (entradillas) } \\
\text { Alineación izquierda } \\
\text { Estilo } \\
\text { Color }\end{array}$ & & $\begin{array}{l}\text { Pocos comunes: } \\
\text { Titular: } \\
\text { Indicar tamaño: } \\
\text { Ausencia: } \\
\text { Ausencia: } \\
\text { Titular: }\end{array}$ & $\begin{array}{l}\text { Serif: S } \\
\text { Subtítulo: } \\
\\
\text { Presencia: } \\
\text { Presencia: } \\
\text { Subtítulo: }\end{array}$ & $\begin{array}{l}\text { serif: } \\
\text { Antetítulo: }\end{array}$ \\
\hline \multicolumn{5}{|l|}{ Multimedia } \\
\hline $\begin{array}{l}\text { Tamaño fotografía principal } \\
\text { Pie de fotografía en portada } \\
\text { Ampliar fotografía en portada } \\
\text { Fotos de otros módulos } \\
\text { Video } \\
\text { Infografía } \\
\text { Audio } \\
\text { Audio directo } \\
\text { Galería } \\
\text { Especiales }\end{array}$ & & $\begin{array}{l}\text { Ausencia: } \\
\text { Ausencia: } \\
\text { Ausencia: } \\
\text { Ausencia: } \\
\text { Ausencia: } \\
\text { Ausencia: } \\
\text { Ausencia: } \\
\text { Ausencia: } \\
\text { Ausencia: }\end{array}$ & $\begin{array}{l}\text { Presencia: } \\
\text { Presencia: } \\
\text { Presencia: } \\
\text { Presencia: } \\
\text { Presencia: } \\
\text { Presencia: } \\
\text { Presencia: } \\
\text { Presencia: } \\
\text { Presencia: }\end{array}$ & \\
\hline \multicolumn{5}{|l|}{ Documentación / Actualización } \\
\hline $\begin{array}{l}\text { Buscador/hemeroteca } \\
\text { Enlaces textuales } \\
\text { Presencia de actualización }\end{array}$ & & $\begin{array}{l}\text { Ausencia: } \\
\text { Ausencia: } \\
\text { Ausencia: }\end{array}$ & $\begin{array}{l}\text { Presencia: } \\
\text { Presencia: } \\
\text { Presencia: }\end{array}$ & \\
\hline \multicolumn{5}{|l|}{ Participación } \\
\hline $\begin{array}{l}\text { Redes sociales } \\
\text { Weblog } \\
\text { Chat } \\
\text { Encuesta } \\
\text { Foro } \\
\text { Entrevistas digitales } \\
\text { Votaciones } \\
\text { Concurso }\end{array}$ & $\begin{array}{l}\text { Ordinal } \\
\text { Ordinal } \\
\text { Ordinal } \\
\text { Ordinal } \\
\text { Ordinal } \\
\text { Ordinal } \\
\text { Ordinal } \\
\text { Ordinal }\end{array}$ & $\begin{array}{l}\text { Ausencia: } \\
\text { Ausencia: } \\
\text { Ausencia: } \\
\text { Ausencia: } \\
\text { Ausencia: } \\
\text { Ausencia: } \\
\text { Ausencia: } \\
\text { Ausencia: }\end{array}$ & $\begin{array}{l}\text { Presencia: } \\
\text { Presencia: } \\
\text { Presencia: } \\
\text { Presencia: } \\
\text { Presencia: } \\
\text { Presencia: } \\
\text { Presencia: } \\
\text { Presencia: }\end{array}$ & Tipo: \\
\hline
\end{tabular}

Fuente: elaboración propia 
El estudio defendido en este artículo se plantea desde el punto de vista del diseño y del contenido. La razón de este doble enfoque se debe, por un lado, a que el diseño también es un mensaje, y existe una vinculación entre lo que transmite y su efecto. En definitiva, todo lo que respecta a la "comunicación visual" (Riffe, 2005:29), que pueda ser "visto e interpretado" (Krippendorff, 2004:13). Está demostrado que los aspectos que influyen en la credibilidad de los cibermedios son el diseño y la estructura visual de la página (Fogg et alt. 2002). Por otro lado, la tecnología es el "centro" del desarrollo del medio (Chisholm, 2010:4); pero coincidimos con Likes (2010:187) en que "el contenido" sigue siendo el núcleo del ejercicio periodístico. De hecho, el contenido se mantiene como referente en las estrategias de futuro definidas por las empresas, ya que pervivirán aquellos medios que aporten un contenido "altamente especializado", bien explicado y contextualizado (Elías, 2010).

Para poder describir la evolución es necesario realizar un análisis cronológico, factor relacionado con la naturaleza del propio análisis de contenido (Hartley, 2004: 26). Se selecciona como fecha desde principio de 2008 hasta junio de 2012. La elección del año 2008 se debe a que en esa etapa se produce un rediseño significativo del site. Para analizar cronológicamente este proceso, se localizan en hemeroteca y se realizan capturas de pantalla de la home del site y de las noticias interiores. Se obtienen, en total, ocho capturas del site y ocho capturas de las noticias interiores. De las aplicaciones móviles, se realizan capturas de todas sus secciones, una por cada sistema operativo analizado, tanto de portada como de noticia.

La ficha de análisis (Tabla 1) está dividida en cinco áreas: estructura, tipografía, multimedia, documentación/actualización y participación. En cada apartado, se añaden las subvariables presencia, ausencia, emplazamiento o tamaño. Estas son las categorías en las que se clasificarán los datos.

\subsection{Justificación de la muestra seleccionada}

La elección del caso de estudio de los40.com se debe a tres factores. El primero, porque los40.com dependen de la cadena de radio líder musical (AIMC, 2012). Segundo, porque es el principal cibermedio musical español con más usuarios (Google Trends, Alexa, AIMC, 2012). Y tercero, porque los 40.com han desarrollado aplicaciones móviles para los sistemas Android, Symbian, e iOS. Es el único medio musical en español con aplicación para iPad (Los40.com, 2010c). Se han seleccionado estos sistemas operativos debido a su mayor implantación en la sociedad: Android (41\%), Symbian (22\%) y iOS (18\%) (Fundación Telefónica, 2011:50). 
Gráfico 1. Medios musicales con más visitas

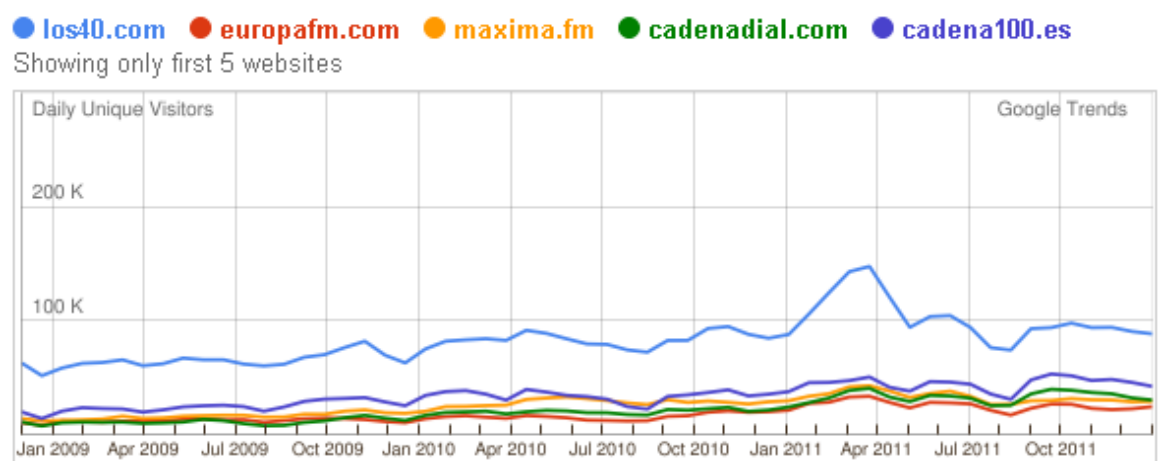

Fuente: Google Trends

Para la cadena 40 Principales, su web funciona como un "punto de encuentro entre los jóvenes y la música". Según datos de Nielsen, aportados por el grupo, el perfil de la web es muy similar entre hombres y mujeres, con un 48\% audiencia universitaria. Más del $72 \%$ de los usuarios de la web son menores de 34 años. En concreto, la audiencia de 12 a 24 años representan el 39\% de los usuarios, de entre 25 y 34 años el 33\%, y de 25 a 49 el $22 \%$ (los40.com, 2009).

La misma franja de edad predomina en el análisis de Alexa (2012) y en Google Trends (2012). Alexa (2012) también aporta otros datos de interés sobre la web, como el hecho de las páginas anteriores y posteriores a las visitas de los40.com suelen ser Google, Facebook, Youtube y diversas redes sociales que, por lo tanto, funcionan como canalizadoras del portal. Cada usuario suele ver, de media, dos páginas de la web, con una media de tres minutos de visita. Según la cadena, al día se producen más de 340.000 aperturas del player, la escucha directa de la radio, cifra que se eleva a más de 10.200.000 aperturas al mes (los40.com, 2011).

Cadena 40, perteneciente al grupo PRISA, fue la primera cadena de emisoras especializadas en música pop que surgió en España, en 1966 (Requejo y Herrera, 2009). Importó el formato Top 40 de Estados Unidos, con una marcada finalidad mercantil (Iñigo, 2002:27). Los40.com se presentó en octubre de 2000 como el portal en español más importante de música (Murelaga, 2007). No obstante, la marca creó dos sitios diferentes, los40.com, y los40principales.com, que al final se unificaron en el primer dominio (Amoedo et alt., 2006: 1). En los comienzos el portal agrupaba a todas las cabeceras musicales del grupo Prisa (elpais.com, 2003b). Desde el principio encabezaron iniciativas enfocadas hacia la descarga legal de música en la red (elpais.com, 2001; 2003a). En 2005, los40.com recibió un premio 
de la Society For News Design. Se detecta un interés por adaptarse con rapidez herramientas y tecnologías incipientes en cada etapa. Por ejemplo, el envío de emails de noticias, mensajes por SMS y boletines en dispositivos como agendas electrónicas y móvil WAP o la introducción de blogs específicos (elpais.com, 2006).

En 2008 se rediseña la web con una comunidad de participación, más vídeos, una sección de videojuegos y se estrenó Internight, programa dedicado a la música y a las nuevas tecnologías, debido a "la proliferación de comunidades digitales (...), una de las aficiones en expansión del target de Los 40 Principales" (elpais.com, 2008). De hecho, en 2007 era la cadena con más oportunidades para la intervención de sus oyentes (Herrera, 2007). Para Requejo y Herrera (2009), "el planteamiento" de los40.com es "el más completo y ambicioso" de radio musical e incluso generalista. Uno de sus éxitos radica en el multimedia. Al mes se emiten 1.100.000 vídeos desde la web (los40.com, 2011). La innovación más reciente es la adaptación a dispositivos móviles como iPhone y iPad (los40.com, 2010). En 2011, dentro de la estrategia de Prisa de distribución multiplataforma, los40.com lanzan aplicaciones para las televisiones LG con acceso a Internet (Europa Press, 2011).

Los 40 Principales se han consolidado años como marca, que también se ha extendido en canales temáticos en televisión y en prensa con Revista 40 (Elpais.com, 2004). En este sentido, Romero y Degrado (2005) critican que el caso de canal 40 TV está limitado al pago de esos contenidos, por lo tanto no se puede apreciar como una televisión que tenga la misma influencia que la radio.

\section{Resultados}

\subsection{Estructura}

La web tiene como base un fondo gris oscuro, sobre el que se inserta una capa blanca, en la que se distribuyen los contenidos de portada. Este tipo de color corporativo se ha mantenido durante el periodo de análisis. Sobre la capa gris se sitúa el logo de la cadena. En 2008 estaba debajo de un módulo de contenidos superior. A partir del rediseño de ese año, se elimina el módulo superior y el logo pasa a estar en primera posición. En todas las muestras analizadas se mantiene un menú de navegación horizontal. El site siempre se ha dividido en dos bloques. El primero sobre actualidad y el segundo con contenidos atemporales. Estos bloques están separados por un módulo, tipo banner, con el fondo gris oscuro, donde se distribuyen contenidos de promoción.

Desde 2008, la zona superior del primer bloque estaba compuesta por seis módulos. Uno central y dinámico rotatorio de contenidos. Rodeando a éste se distribuían los cinco módulos 
fijos restantes. El módulo dinámico de imágenes y el texto anuncian contenidos de forma automática, aunque el usuario también puede controlar la navegación por este menú. Bajo este módulo se desplegaba en las portadas, desde 2008, una estructura vertical dividida en tres columnas, cada una estaba destinada a un contenido específico. Esta estructura se modifica a finales de 2011, donde las columnas pasan a ser filas. Es decir, se pasa de una estructura vertical a una horizontal. Desde entonces, el módulo superior se divide en dos zonas. Una dedicada completamente al módulo dinámico rotatorio, que ya no está rodeado por otros contenidos. Y la segunda zona, un módulo dividido a su vez en seis partes, con fotos y titulares informativos. Debajo de este espacio surgen tres filas, cada una destinada a un contenido específico, y en ellas se agrupan cinco noticias, una de tamaño mayor, que abre la sección y cuatro de tamaño menor. El segundo bloque de contenidos ha estado dividido en columnas desde 2008 hasta finales de 2011, donde ahora también se ha optado por una distribución.

La estructura de las noticias no ha tenido cambios visuales en el periodo analizado. Empieza distribuida a dos columnas en el titular y a tres columnas en el resto de la información, cada columna dedicada a un contenido.

Las aplicaciones para móvil de Android y Symbian se estructuran en un menú de navegación con tres botones en la zona inferior: En directo, Lista 40 y Noticias. En la zona superior se sitúa el logo de los40.com, con fondo en color negro. La aplicación para iPhone tiene un diseño similar a las anteriores, pero con prestaciones diferentes. El color de fondo es gris. La estructura es igual a la anterior aplicación, pero con una sección más; Game 40, donde se ofrecen los principales juegos disponibles para su descarga. Igualmente, la sección de la Lista de Los 40 Principales permite la compra de canciones en mp3.

En el iPad, la amplitud de la pantalla permite una reconfiguración de la estructura. En la zona superior izquierda se sitúa el logo, y el menú de contenidos pasa a la zona superior derecha, similar al menú de navegación del site. Se disponen dos columnas. A la izquierda, en color de fondo negro y más estrecha, está siempre disponible un acceso a la escucha online. Bajo el player, se dispone un módulo con información del programa que se emite en ese momento. A la derecha se despliega la segunda columna más ancha, con fondo gris. En ella se abre el contenido de cada una de las secciones. 
Ilustración 1. Capturas de pantallas del site y de aplicaciones móviles

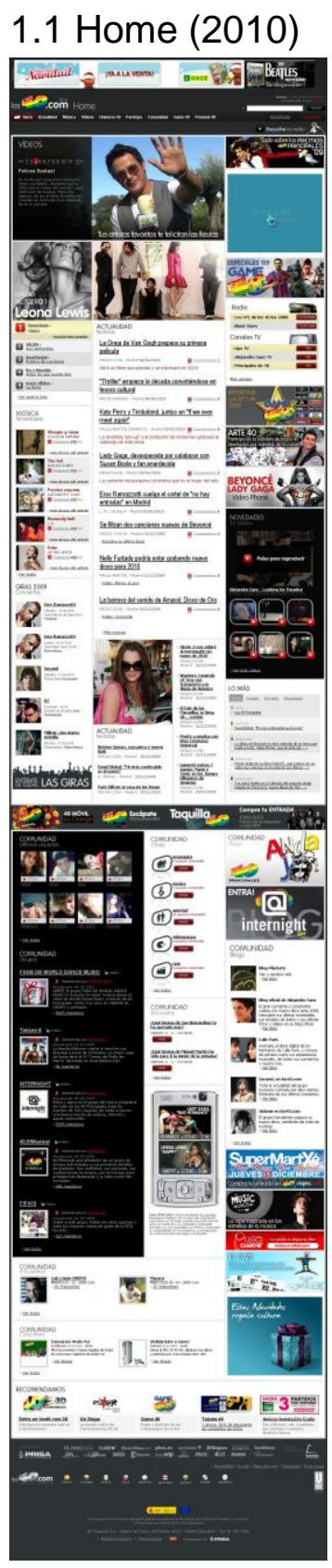

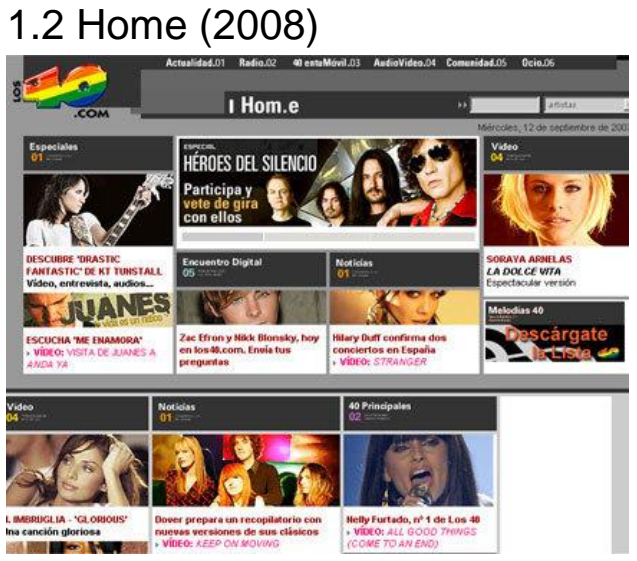

1.4. App iPhone (2011)

\subsection{Home (2009)}

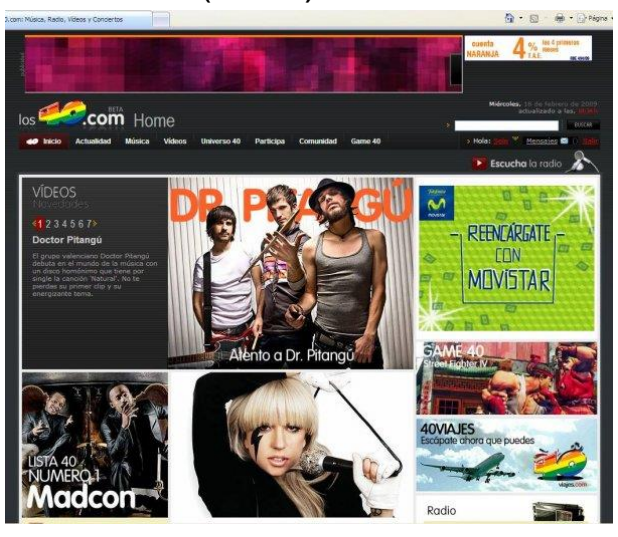

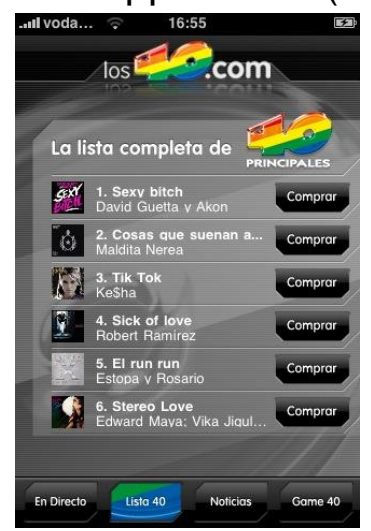

\subsection{App iPhone (2012)}
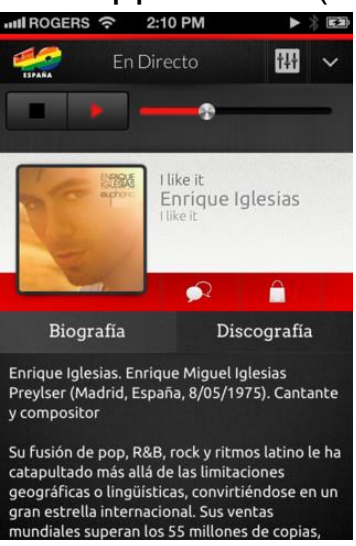

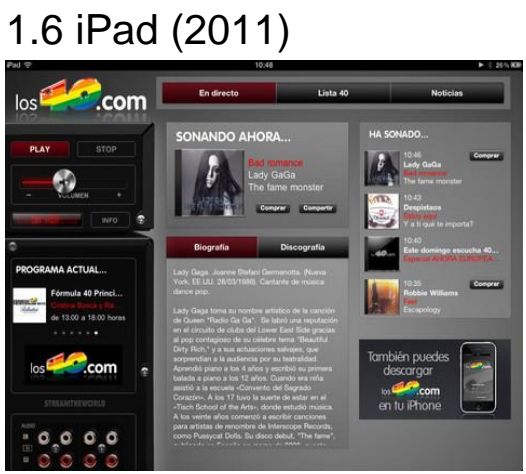

1.7 iPad (2012)

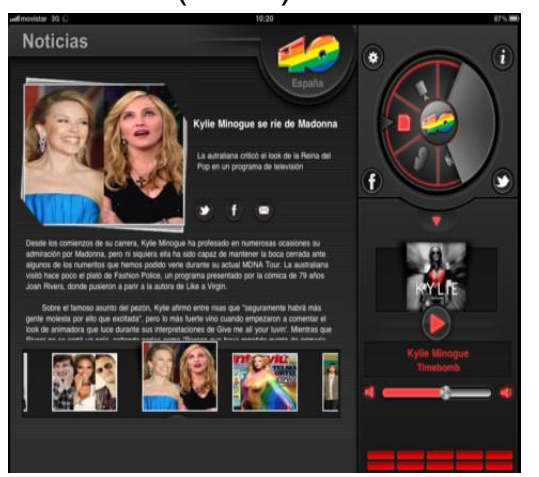

Fuente: elaboración propia

Facultad de Ciencias de la Información - Universidad de La Laguna

Avenida César Manrique, s/n; Campus de Guajara

38071 La Laguna, Tenerife (Islas Canarias - España) 


\subsection{Tipografía}

La tipografía, en todos los casos analizados, tanto en portada como noticia, siempre ha sido sans serif, con uso de Arial negrita para el texto que va sobre imágenes, Arial Narrow para los titulares, y Tahoma para los subtítulos o los textos. En las noticias, el titular se distribuye a dos columnas, y después, se pasa a tres: las de los laterales con contenido multimedia, y la central, a una columna, la información que, de media, no supera entre las 8 (el más breve) y las 30 líneas (el más extenso). También se separan los textos por espacios en blanco, que aportan claridad.

En la aplicación móvil de Android, la tipografía es sans serif. Los titulares tienen todos al mismo cuerpo, por lo tanto no se produce una diferenciación visual de importancia de la información, salvo por el orden del emplazamiento. Dentro de la noticia, el titular tiene un cuerpo mayor que el subtitular, en estilo cursiva. Los textos son los mismos que los del cibermedio. Para la aplicación de Symbian el menú de noticias, aunque conserva su estructura horizontal sin diferencias en el cuerpo de los titulares, incluye una pequeña fotografía a la izquierda, titular en color blanco y dos líneas como subtítulo. Cuando se pulsa en el enlace Más, se accede directamente a la noticia publicada en los 40.com, al navegador. Por lo tanto, no se realiza una adaptación visual del contenido.

En la aplicación móvil de iPhone, la tipografía también es sans serif y el fondo gris. Los titulares del menú de las noticias son en color blanco, con el mismo cuerpo. En el caso del ¡Pad, la disposición de las noticias, en scroll, sigue en disposición horizontal, por filas, pero aportan más datos. Cada una tiene un titular en blanco, subtítulo rojo y un antetítulo donde aparece la firma (autor) y hora de publicación.

\subsection{Multimedia}

En el análisis cronológico se detecta un aumento de la cantidad de fotografías y de su tamaño. Si bien en las primeras portadas de 2008 el módulo superior incluía fotografías, éstas eran más horizontales y estaban rodeadas por unas cajas grises que indicaban la sección y el titular. A partir del rediseño de 2008, esas fotografías se hacen más grandes aprovechando el espacio de esas cajas e incluyendo la sección y el titular dentro de la misma imagen. La fotografía pasa de 190×94 a 198 x 198. Igualmente, el módulo dinámico se amplía a un tamaño de $667 \times 330$. También existe un cambio de cantidad. De las 25-30 fotografías que había en portada en 2008, se pasa a unas 35-40 imágenes en el último diseño de finales de 2011, por lo que aumentan los centros de impacto. En las noticias interiores se potencia también la imagen con cinco centros de impacto visual. Una imagen a la derecha, de 310×310, con opción de ampliación. A la izquierda, otra imagen resaltada por 
un módulo con fondo negro, la ficha del artista y el apoyo multimedia de un videoclip y una fotogalería.

En las noticias interiores, la columna de la derecha se dedica a una fotografía, que puede ampliarse, publicidad y el ranking de los contenidos más vistos. La columna izquierda también está protagonizada por la imagen más pequeña del protagonista de la información, fotografía que ilustra un módulo que permite el acceso a la ficha del artista, visualización directa de un vídeo, acceso a un menú de seis vídeos, enlaces a noticias relacionadas y fotogalería.

En 2011, en la aplicación móvil de Android, Symbian e iPhone, En directo consiste en un sencillo player con adaptador de volumen y un espacio donde se indica el cantante que se escucha en ese momento, una fotografía, y título de la canción. La Lista de Los 40 Principales se distribuye en una tabla ordenada, fotografía del álbum y título. La sección de noticias está compuesta por un menú de veinte titulares, sin fotografía, situados uno debajo de otros en filas. En las noticias, siempre introducen una fotografía, a una columna, como el texto, que separa el titular de la información. Para la aplicación de Symbian, sin embargo, las noticias pierden su configuración de aplicación. En el iPad, se mantienen fotografías en las secciones de radio online y en la lista de éxitos. Sin embargo, no se añaden enlaces a vídeo ni fotogalerías en las noticias, ni aparecen fotografías en el menú de acceso a esas noticias, solo una imagen ya en su interior. Ya en la nueva versión de 2012 , en el iPad, se añade un sistema de navegación rotatorio y se amplían las secciones. La navegación entre noticias se mantiene a través de un carrusel de fotografías.

\subsection{Contenidos}

En el primer bloque de la estructura, mientras en la portada de principios de 2008 el enlace directo a la escucha online pasaba desapercibido en el botón player situado en la parte superior; a partir del rediseño de ese mismo año, se da un mayor protagonismo a este servicio. Desde entonces, se sitúa justo debajo del menú de navegación, con un enlace directo a la señal de radio. El enlace está identificado por un botón de player y el texto "Escucha la radio", acompañado de la imagen de un micrófono. En esta frase, la palabra "escucha" está marcada en negrita. A partir del último rediseño de finales de 2011 , la imagen del micrófono desaparece para sustituirse por el logo de Los 40 Principales y se añade la opción de "y Participa", incluyendo a la derecha un enlace directo al perfil de la empresa en Facebook. Los contenidos generales de la web son Actualidad, Música, Vídeos, y la Lista de Los 40 Principales. No obstante, se han producido cambios de emplazamiento de determinados contenidos, se han añadido nuevos y se han suprimido o relegado otros en el periodo analizado. 
El último rediseño realizado a finales de 2011, estructurado en filas, ha provocado una nueva situación de los contenidos. Mientras desde 2008 hasta finales de 2011 la columna derecha estaba destinada, principalmente, a contenidos de promoción (canales de radio o televisión específicos de los40.com) o publicidad, el diseño posterior ha modificado este contenido por un espacio destinado a las redes sociales y por la Lista de Los 40 Principales, que desde 2008 había permanecido en la columna izquierda. En cuanto a la Lista de Los 40 Principales, desde este menú se accede a una ficha del cantante, y a la canción clasificada. Si se pulsa en esta opción, se permite la escucha completa de la canción, ver el videoclip o comprarla por iTunes. Antes de 2011, solo se permitía oír 30 segundos de las canciones y se exigía un registro al usuario si deseaba escucharla completa. De la columna derecha se ha suprimido la sección de vídeos (que estaba ahí desde 2009) y el ranking de los contenidos más vistos, votados, enviados y escuchados, al que ahora solo se accede desde las noticias interiores.

Hasta finales de 2011 la columna central se dedicaba a contenidos puramente informativos. Ahora, con la nueva disposición horizontal, los contenidos se estructuran en cuatro filas temáticas: Destacados, Noticias, Programas y Tendencias. De esta forma, los programas de la cadena, cuya información siempre estaban en el segundo bloque, aumentan de categoría en su emplazamiento.

El segundo bloque de la web ha estado dedicado, hasta finales de 2011, a la participación. Sin embargo, este contenido se ha reducido al fortalecer la presencia de las redes sociales, emplazadas en la zona superior derecha de la web. Desde la fecha indicada se ha potenciado este bloque como zona multimedia, con el acceso de un listado de videoclips destacados, y datos sobre giras. Únicamente, relacionado con la Comunidad, se han dejado las secciones de Encuestas, Encuentros y Concursos. Antes de este rediseño, la columna izquierda, dedicada a la sección Comunidad, estaba con enlaces y fotografías de algunos de los usuarios, así como de los grupos creados en su comunidad. Era el único contenido de participación que se destacaba sobre un fondo negro, ya que era un espacio único creado por el medio. La columna central estaba dedicada a los chats (con temáticas sobre programas, música, amistad, videojuegos y cine) y la derecha a los blogs de programas y artistas. En la parte inferior de las columnas se distribuían otros contenidos, como videochats, concursos o encuestas.

El menú estático es el organizador del contenido de la web. En 2008, las secciones de este menú eran Actualidad, Radio, EntuMóvil, AudioVídeo, Comunidad y Ocio. En el nuevo diseño de 2008 se cambiaron a Actualidad, Música, Vídeos, Universo 40, Participa, Comunidad y Game 40. Es decir, los contenidos audiovisuales se separaron para crear unidades autónomas e independientes. Universo 40 reunía aquellos contenidos creados por la marca. 
En la sección Game 40 se ofrecían contenidos relativos al sector del videojuego, separados por dispositivos, además de noticias y un apartado en vídeo con acceso a tráiler, programas de televisión dedicado a las novedades del videojuego y a podcast procedentes de las emisiones en radio. Ahora a estos contenidos se acceden desde una web independiente, www.game40.com.

A finales de 2011 se modifica de nuevo este menú suprimiendo las opciones de Universo 40 y Game 40 para dejar los siguientes contenidos: Actualidad, Música, Vídeos, Tendencias, Participa, Comunidad, Premios 40, Revista 40, Elplan.tv (Ballantines). El apartado de actualidad integra la información referente a noticias, especiales, fotogalerías, giras 40, festivales, y blogs. En la sección música se incluye un acceso a artistas, listas, los canales de radio (que integran ocho canales especializados por estilos), y novedades discográficas. En vídeos, el usuario tiene acceso a siete canales de tv creados por los40.com. Tendencias aglutina Noticias, Moda, Libros, Belleza y Tecnología. Aquí se integran las noticias de videojuegos. En la sección Participa se incluyen las herramientas interactivas que permiten la relación entre los usuarios y el medio, como los encuentros, el chat, las encuestas, los foros, los concursos, y sugerencias. En Comunidad, la red propia creada por la cadena, se exige un registro al usuario. Premios 40 es una sección especial dedicada a los contenidos generados en la ceremonia que anualmente se celebra con motivo de estos galardones. Revista 40 ofrece en abierto contenidos de la revista impresa. Planb.tv es un proyecto de concurso musical patrocinado por una empresa de bebidas alcohólicas.

Los contenidos de la aplicación móvil en Android, Symbian e iOS se limitaban en 2011 a la escucha online, la lista de éxitos y las noticias de actualidad. No se podía oír canciones, ni ver videoclips. Los contenidos para iPad eran los mismos, excepto la sección Game 40, que no estaba incluido. En la Lista de Los 40 Principales se permitía la compra de los mp3, tanto para iPhone como en iPad. En la nueva versión de 2012, se añade la opción de ver vídeos y escuchar canciones. Igualmente, se ha potenciado un multiacceso a contenidos de los demás sites de los40.com e Latinoamérica y, con un nuevo menú desplegable en los iPhone, Android y Symbian se acceden a más secciones: En Directo, Lista 40, Noticias, Audios 40, Videoclips, Vídeos 40, Programación y Países. Los videoclips no se integran en la aplicación, sino que redireccionan a Youtube.

\subsection{Documentación / Actualización}

En el site de los40.com se añade la función de búsqueda de contenidos. Sin embargo, carece de una hemeroteca digital que permita realizar búsquedas más precisas. No obstante, las noticias incluyen enlaces que remiten a informaciones previas, bien dentro del propio texto o bien en un módulo añadido a la noticia, donde se completan con enlaces con noticias 
publicadas anteriormente. Igualmente, cada noticia añade siempre un enlace a un audio y a un vídeo del artista. Tanto la portada como las noticias incluyen la fecha y hora de actualización, en color rojo.

En las aplicaciones de Android, Symbian e iOS (iPhone-iPad) no existe opción de búsqueda de contenidos ni hemeroteca. Igualmente, desaparecen los enlaces que remiten a otras informaciones, ni textuales, ni de audio ni de vídeo, excepto y en la biografía y discografía del artista en la sección En Directo; y en el caso de Symbian, cuyos contenidos remitían a la noticia del site original. No se indica actualización de contenidos en estas aplicaciones, excepto para iPad, donde al lado de cada titular sí se especifica la fecha y hora de la publicación de la información.

\subsection{Participación}

Las herramientas de participación, como la Comunidad, siempre estuvieron emplazadas en el segundo bloque de portada. A finales de 2011, la importancia de las redes sociales ha llevado a que se le otorgue menos relevancia a este canal exclusivo de la cadena. Las redes sociales pasaron a ser uno de los pilares clave y se situaron en la zona superior de portada, junto al módulo dinámico y justo debajo del enlace a uno de los contenidos más solicitados de la cadena, la escucha online. De nuevo, quedó reforzada visualmente la emisión online y la participación de los oyentes. Bajo el titular "Síguenos en redes sociales" se añadió un botón a cada una de ellas: Facebook, Twitter, Tuenti y Youtube. En 2012, ese espacio ha sido sustituido por el enlace a los blogs de la cadena. En 2008, y 2009 se invitaba a distribuir el contenido por otras redes como Delicious, My Space, Yahoo, o Menéame. Estas opciones se han retirado y se han incluido como opción de compartir, directamente, a través de Facebook, Twitter o Google+.

En 2011 no existía participación a través de redes sociales desde la aplicación móvil para Android, Symbian y iOS. Sin embargo, en la de 2012, sí se añaden botones para compartir por las redes Facebook y Twitter, tanto en el menú de acceso como en las noticias de interior.

\section{Discusión}

La estrategia digital de Los 40 Principales ha estado encaminada al fenómeno de pantallización de la sociedad, como manifestaba De las Heras, al integrar su portada en otros dispositivos móviles, en relación con el crecimiento experimentado por los smartphones, y la llegada de las tabletas (iPad); además de adaptar su revista impresa al site. Esta opción 
responde a la necesidad del alto nivel de consumo de contenidos digitales que realizan los jóvenes, como citaba el informe de la Fundación Telefónica.

Desde 2008 se ha producido un rediseño progresivo, con una mayor potenciación de los componentes audiovisuales y se ha adaptado a los hábitos sociales juveniles. En el primer caso, su página de inicio se caracteriza por su impacto visual. Las fotografías han adquirido un mayor peso en la composición de la estructura, tanto en tamaño como en cantidad. Junto a las fotografías, el vídeo es uno de los componentes fundamentales de esta web, que actúa como canal de difusión del género audiovisual propio del la música, los videoclips. La crítica de ver determinados contenidos sólo a través de la plataforma de Digital +, como mencionaban Romero y Degrado, queda en parte subsanada por la emisión de vídeos en la red. La potenciación de este componente audiovisual, el mayor tamaño de los titulares, la presencia de textos breves con tipografía sans serif y la documentación de las informaciones coinciden con las preferencias de los usuarios mostradas por Bernal y el Poynter Institute.

En el segundo caso, se ha reconfigurado la participación en el portal en función de las tendencias producidas. Como citaban García, Bringué y Sádaba, las redes sociales ayudan a reafirmar la identidad de grupo, y la comunidad privada creada por la cadena permite reforzar el sentimiento musical. Igualmente, se observa por parte de Los 40 Principales un interés por adaptar el uso de redes a los hábitos actuales, de forma que en el tiempo analizado en el site se ha pasado de ofrecer la opción de compartir en diversas redes sociales a limitarlas a las más usadas, como contemplaba Sánchez. Igualmente, las redes sociales elegidas son las que, de acuerdo con los datos de Alexa, impulsan a priori el acceso al site de la cadena. La creación primera de la Comunidad, en el año 2008, permitió a la empresa realizar una recolección de datos sobre sus usuarios afiliados, conociendo mejor el perfil de su audiencia.

Han fortalecido paulatinamente el acceso a la escucha online de la programación, uno de los servicios más empleados por los usuarios, según datos de la cadena musical. Está situado en un lugar estratégico del sitio, en la zona superior de la página, con una tipografía mayor que los otros elementos que le rodean. Este uso se justifica también con la tendencia a realizar un consumo simultáneo de medios, como anunciaba Fumero (2007). Igualmente, al lado de este acceso y debajo, un módulo recuerda la participación en las redes sociales, fomentando esta tendencia citada. Junto a este servicio se ha reforzado en el diseño, otorgándole un mejor emplazamiento, otro de los contenidos más solicitados según los datos de Google y Alexa; la Lista de Los 40 Principales. En el site se da la opción de escucha y de descarga legal de los contenidos, en línea a la propuesta defendida por Sedeño. La web fomentó durante dos años la sección de videojuegos, pero ésta se ha trasladado a la sección de Tecnología. 
La propia cadena potencia en la web su imagen de marca, como una estrategia de marketing, ofreciendo servicios destinados al ocio que cuentan con el sello de Los 40 Principales, como gestor de viajes, y venta de entradas de conciertos.

En las aplicaciones para smartphone e iPad se detecta un interés por fomentar la movilidad y portabilidad de sus contenidos. Por esta razón, se incluye como servicio digital la escucha en directo de la programación de la cadena. La aplicación para el sistema Android y Symbian es útil y ágil en cuanto permite su función principal, la movilidad de los contenidos. Las deficiencias detectadas en las aplicaciones en 2011 (mayor adaptación audiovisual de la aplicación, acceso a videoclips, formas alternativas de ingresos, como el acceso a iTunes que se ofrece en la web y; sobre todo, la posibilidad de compartir las experiencias de lectura o de escucha con las redes sociales) han sido subsanadas en la versión de 2012. Respecto a Symbian es criticable que el contenido de las noticias remite al enlace directo del navegador, sin producirse una adaptación formal al soporte que facilite la lectura. En iPhone e iPad sí existe una adaptación, y presentan un mayor cuidado formal en la composición visual. Igualmente, se permite un acceso a la compra de contenidos digitales de forma legal, tanto de música (iPad) como de música y videojuegos (iPhone).

\section{Conclusiones}

Respecto al objetivo A se ha producido una evolución del site los40.com encaminado a fortalecer el multimedia y la participación de los usuarios. Se mantienen constantes los colores corporativos del medio desde 2008 hasta 2012 y se ha pasado de un diseño vertical a uno horizontal, con una temática más diversa. Se han conservado los dos bloques que componen el site, tanto de actualidad como de contenidos atemporales. En todos los casos se mantienen como rasgos formales los fondos en blanco, la tipografía en sans serif, la documentación de hipertexto audiovisual, los textos breves de las noticias y una alta potenciación de las fotografías, tanto en cantidad como en tamaño. En la portada y en las noticias se destaca la actualización.

Cadena 40 ha realizado una adaptación más completa del site a los dispositivos móviles, con una ampliación de contenidos. No obstante, en todos se destacan cuatro pilares: la escucha de la radio online, la Lista de Los 40 Principales, la publicación de noticias y el acceso a vídeos. Las aplicaciones conservan los rasgos formales del site, con la composición cromática de gris, blanco y negro; con la presencia de imágenes y el mantenimiento de la tipografía sans serif. Sin embargo, en las noticias los textos son los mismos de la edición del site, y no se ofrecen enlaces a otro tipo de contenidos, ni textuales ni multimedia. Hasta la 
versión de 2012, no se han creado espacios destinados a compartir contenidos en las redes sociales. No obstante, se evidencia una aplicación más desarrollada en iOS, y menos para Android y Symbian, sobre todo. Salvo la utilidad de la escucha online, estas aplicaciones constituyen en la sección de noticias un mero traslado de contenidos de actualidad de la web.

Respecto al objetivo $\mathrm{C}$ se ha detectado una evolución de servicios y contenidos, potenciando o disminuyendo otros durante el tiempo analizado. Como la creación de blogs y de comunidades de participación; que han sido solapados por la presencia de las redes sociales. Actualmente, el multimedia, con la creación de canales online de vídeos por temáticas, y la participación son los pilares básicos de su estructura, como respuesta a las características de la red más demandadas entre los jóvenes. El acceso directo a la escucha de la radio en la página principal evidencia la tendencia de sus usuarios de realizar dos consumos simultáneos. La decisión de incluir como inicio la escucha online en las aplicaciones evidencia el interés por fomentar la movilidad de la radio y de los contenidos del site.

En resumen, el site de los40.com es un ejemplo de convergencia de las cualidades del periodismo impreso y audiovisual, unifica necesidades musicales de la juventud y fomenta su función social como canal de comunicación entre sus usuarios. La música, al ser su componente básico, ha sido potenciada en todo el sitio con el desarrollo de secciones que permiten la escucha y visionado de contenidos digitales musicales, como los videoclips. Los jóvenes parecen haber superado la seducción tecnológica para fomentar su función social, como canal de ocio y de comunicación con los demás; de ahí el interés de la cadena por potenciar los enlaces de participación en las redes sociales, que a su vez actúan como un nuevo cauce de distribución. Se ha producido una evolución de los contenidos, adaptándose e integrando nuevas secciones en función del desarrollo de la industria de contenidos digitales. En los dispositivos móviles, se detecta una distribución multiplataforma con adaptación formal, pero no de contenido. Sí destacan, en todas estas aplicaciones, su valor de movilidad a través de la escucha online desde los dispositivos y su potenciación de las redes sociales y el multimedia.

Este artículo pretende servir de punto de partida para futuras investigaciones, necesarias para conocer mejor a la audiencia juvenil y adaptar a los cibermedios, en los próximos años, a las necesidades y expectativas que estas generaciones han experimentado en el consumo de información en la red. 


\section{Bibliografía}

Adorno, T. (1973): El arte en la sociedad industrial. Buenos Aires: Rodolfo Alonso.

AIMC (2012): Estudio General de Medios. En: www.aimc.es.

Alexa (2012): Ranking los40.com. Spain.

Amoedo, A. et alt. (2006): “Estrategias de comunicación de las cadenas musicales comerciales españolas en la red: análisis de los40.com, cadena100.es, europafm.es y kissfm.es", en XXI Congreso Internacional de Comunicación CICOM: Los jóvenes y el nuevo escenario de la comunicación. Pamplona, Facultad de Comunicación.

Armentia, (2007): "Últimas tendencias en el diseño de los ciberdiarios españoles". Congreso de Periodismo Digital. Presente y futuro de la comunicación digital. En: http://www.congresoperiodismo.com/.

Atton, C. (2009); "Writing about listening: alternative discourses in rock journalism", Popular Music, no 28, Cambridge University Press, pp. 53-67.

Bernal, A. y Cabrera, M. A. (2011): Identidad y desarrollo de un portal femenino en la red. Estudio de caso de Hola.com. III Congreso Internacional Latina de Comunicación Social. "La comunicación pública, secuestrada por el mercado". Universidad de La Laguna, Tenerife.

Bernal, A.I. (2009): Los nuevos medios de comunicación y los jóvenes. Madrid: Euroeditions.

Brenna, M. (2005): Writing to reach you: the consumer music press and music journalism in the UK and Australia.PhD thesis, Queensland University of Technology. http://eprints.qut.edu.au/16141/

Bringué y Sádaba, C. (2009): La generación interaciva en España. Niños y adolescentes ante las pantallas. Madrid: Ariel, Fundación Telefónica.

Burset, S. y Sánchez, L. (2009): "Fotologs y adolescentes: identidad imagen y texto en pantalla", Doxa Comunicación, no8, pp. 117-144.

Cabrera, M. (2009): "El diseño de la prensa digital española en el contexto de la convergencia tecnológica. La identidad visual del ciberperiodismo", Revista Latina de Comunicación Social, nº 64, pp. 766-786.

Calvi, J. (2006): "La industria de la música, nuevas tecnologías digitales e Internet. Algunas transformaciones y salto en la concentración”, Zer, no 21, pp. 121-137.

Canga, J. (2005): "Periodismo en la Red. Diseño periodístico y ediciones digitales", Telos: Cuadernos de Comunicación e Innovación, nº 63, pp. 71-76.

Change Wave Research (2011): Consumer Tablets and eReaders - iPad Momentum Continues. Rockville, Maryland. 
Chisholm, J. (2010): "The future is in the hands of journalist", British Journalism Review, nำ, pp.13-19.

Díaz Noci etl atl. (2010): "Convergencia y contenidos", en López, X., Pereira, X. (coord.). Convergencia digital. Reconfiguración de los Medios de Comunicación en España. Santiago de Compostela: Universidad Santiago de Compostela, pp. 167-188.

Elías, C. (2010): "The Future of Journalism in the Online Public Sphere: When Journalistic Sources Become Mass Media in their Own Right", Estudios sobre el Mensaje Periodístico, no 16 , pp. 45-58.

Elpais.com (2001): "Los internautas descargan 30.000 canciones". En: http://www.elpais.com/articulo/espectaculos/internautas/descargan/30000/canciones/ofert a/Gran/Via/Musical/elpepiesp/20010120elpepiesp_8/Tes

Elpais.com (2003a): "Nuevo servicio de descargas de música en los40.com". En: http://www.elpais.com/articulo/internet/los40/com/Nuevo/servicio/descargas/musica/los40/ com/elpeputec/20030322elpepunet_1/Tes

Elpais.com (2003b): "Los40.com celebra su primer aniversario". En: http://www.elpais.com/articulo/cultura/los40/com/celebra/primer/aniversario/millones/visita s/mensuales/elpepucul/20030701elpepucul_1/Tes

Elpais.com (2004): "Nace Revista 40, referente de música y ocio para los jóvenes”. En: http://www.elpais.com/articulo/sociedad/Nace/Revista/referente/musica/ocio/jovenes/elpe pisoc/20040922elpepisoc_12/Tes

Elpais.com (2006). "Los40.com estrena blogs de LOVG, Coti y Ana Torroja”. En: http://www.elpais.com/articulo/cultura/Los40/com/estrena/blogs/Oreja/Van/Gogh/Coti/Ana/ Torroja/elpepucul/20060804elpepucul_2/Tes

Elpais.com (2008): "Los 40 Principales estrenan 'Internight". En: http://www.elpais.com/articulo/gente/Principales/estrena/Internight/elpepugen/20080915el pepuage_2/Tes.

Elpais.com (2010): "MTV arranca sus emisiones en abierto por TDT". En: http://www.elpais.com/articulo/Pantallas/MTV/arranca/emisiones/abierto/TDT/elpepurtv/20 100916elpepirtv_5/Tes

Elpais.com (2011): "Super Pop deja de publicarse en papel". En: http://www.elpais.com/articulo/sociedad/Super/POP/deja/publicarse/papel/elpepusoc/2011 0510elpepusoc_6/Tes

Europa Press (2011): "El País, As y Los40 lanzan aplicaciones para televisiones LG con acceso a Internet", en eleconomista.es, 2 de agosto de 2011.

Eye Square (2010): iPad -the new way of reading: Goodbye Gutenberg. Germany: Eyes Square $\mathrm{GmbH}$, noviembre. 
Fogg, B.J., et alt. (2002): How the people evaluate the web site's credibility? Results from a large study. Persuasive Technology Lab, University of Stanford.

Forde, E. (2001): "From polyglottism to branding: On the decline of personality journalism in the British music press", Journalism, ํㅡ 2, pp. 23-43.

Franquet, R. et al. (2007): "La información en los principales medios de comunicación online. Estudiar la representación de género", Zer, no 22, pp. 267-282.

Fumero, A. et alt (2007): Web 2.0. Madrid: Fundación Orange.

Fundación Telefónica (2011): La Sociedad de la Información en España 2010. Madrid: Ariel, Fundación Telefónica.

Fundación Telefónica (2012): La Sociedad de la Información en España 2011. Madrid: Ariel, Fundación Telefónica.

Funes, M. (2008): "Cultura, Política y Sociedad”, en López, A. (Coord.): Informe Juventud en España 2008. Madrid: INJUVE.

Galindo, F. (2008): "Implicación tecnológica del audiovisual para dispositivos móviles. Estado de la cuestión y propuesta de modelos de producción”, Doxa Comunicación, nำ, pp. 113-138.

García, A. (2010): "Radio digital e interactiva. Formatos y prácticas sociales", Revista Icono14, no 15 . pp. 133-146.

Gimeno, M. (dir.) (2011): Informe anual sobre el desarrollo de la sociedad de la información en España. Madrid: Fundación Orange.

Goggin, G. (2010): Global Mobile Media. New York-Oxon: Taylor and Francis.

Google Trends (2012). Tráfico los40.com

Gymnich, M. et alt. (2010): Gendered (Re)Visions: Constructions of Gender in Audiovisual Media, Gottigen: V\&R Unipress, Bonn University.

Hartley, J. (2004): "Case study research", en Cassell, C. y Symon, G., Essential guide qualitative methods in organizational research, London: Sage, pp. 323-333.

Hernández, R. et alt. (2006): Metodología de la investigación. Madrid: Mc Graw Hill.

Herrera, S. (2007): "Las nuevas modalidades para la participación de los jóvenes en la radio española", Trípodos, $\mathrm{n} \cong 20$, pp. 171-188.

Holt, Fabian (2011). "Is music becoming more visual? Online video content in the music industry. Visual Studies, vol. 26, no 1, pp. 50-61

Hormigos, J., y Martín, A. (2004): "La construcción de la identidad juvenil a través de la música", Revista Española de Sociología, oㅡ 4, pp. 259-270.

Igartua, J. (2004): Teoría e investigación en comunicación social. Madrid : Síntesis 
INE (2011): Encuesta sobre equipamiento y uso de tecnologías de la información y comunicación en los hogares. Madrid: Inebase.

Iñigo, J. (2002): "Orígenes de la radio musical en España", Espacios Hispanoamericanos, no 630 , pp. 25-35.

Jensen, Klaus y Larsen, Peter (2010). "The Sounds of Change: Representations of_Music_in European Newspapers 1960-2000”, en Gripsrud, Jostein y Weibull, Lennart Media, Markets and Public Spheres: European Media at the Crossroads, Bristol, UK ; Chicago, IL : Intellect, 2010, pp. 249-265.

Jones, Steve (2002). "Music that moves: popular music, distribution and network technologies, Cultural Studies, vol. 16, no 2, pp.213-232.

Kohlbacher, F. (2006): "The use of qualitative content analysis in case study research", Forum: Qualitative social research, vol.7, no 1, pp.1-38.

Krippendorff, K. (2004): Content analysis. A introduction to its methodology, London: Sage Publications.

Lawson, K. (2010): Reinventing journalism: using mobile technology to change the media. Australia: RMIT University.

Likes, T. (2010): "The future of TV News: Is technology outpacing journalistic standards?”, Electronic News, ํㅡㄴ, pp.184-187.

López, N. (2011): “Jóvenes y televisión en 2010. Un cambio de ámbitos", Zer, no 30, págs. 97-113.

los40.com (2005): "Los40.com recibe dos galardones de Society of News Design". En:

los40.com (2008): "Bienvenidos al nuevo portal de los40.com". En: http://www.los40.com/actualidad/noticias/bienvenidos-al-nuevo-portal-delos40com/nota/1292251.aspx.

los40.com (2009): Datos de audiencia, http://www.los40.com/static/publicidad.html [fecha de consulta: 28 de diciembre de 2009]

los40.com (2010): "Los40.com lanza su aplicación para iPhone, iTouch e iPad". En: http://www.los40.com/actualidad/noticias/los40com-lanza-su-nueva-aplicacion-paraiphone-itouch-e-ipad/nota/1302995.aspx

los40.com (2011): Datos de audiencia. En: http://www.los40.com/publicidad.aspx.

Megías, I. y Rodríguez, E. (2003): Jóvenes entre sonidos: hábitos, gustos y referentes musicales. Madrid, INJUVE y Fundación de Ayuda contra la Drogadicción (FAD).

Meier, C. (2011): Erlösmodelle im E-Publishing: Wie sich Medien auf Tablets und Smartphones neu erfinden Könnnen. Hamburg: Kress. 
Murelaga, J. (2007): "Reflexiones sobre la transformación del concepto 'programación radiofónica’. De la parrilla al mensaje”, Palabra clave, $n^{\circ}$ 2, p. 113-124

Naval, C. y Sádaba, C. (2005): "Juventud y medios de comunicación frente a frente", Revista Juventud, no 68.

Navarro, L. (2011): La industria de los contenidos en Internet”, Question, 1, pp. 1-6.

Nielsen Wire (2010): "Young and Mobile: A Global View of Cellphones and Youth", Nueva York: Nielsen.

Nielsen, J.. (2001): Usabilidad. Diseño de sitios Web. Madrid : Prentice Hall.

Ontsi (2011): Las redes sociales en Internet 2011. Madrid: Onsti, Observatorio Nacional de las Telecomunicaciones y de la SI.

Paschal Preston, Jim Rogers, (2011) "Social networks, legal innovations and the "new" music industry", info, Vol. 13 Iss: 6, pp.8 - 19

Pino, C. y Galán, E. (2010): Jóvenes, ficción televisiva y nuevas tecnologías. Área Abierta, nำ 25 .

Quirk, T. y Toynbee, J. (2005): "Going through the motions: popular music performance in journalism and in academic discourse", Popular Music, no 24 , Cambridge University Press, pp 399-413.

Requejo, J. y Herrera, S. (2009): "Los nuevos recursos participativos de la Web 1.0 y 2.0 en los portales de las emisoras musicales dirigidas a jóvenes", Comunicación y hombre, $\mathrm{n}$ o 5, pp. 23-40.

Riffe, D. et alt. (2005): Analyzing media messages: using quantitative content analysis in research. Mahwah, N.J: Lawrence Erlbaum.

Rodríguez de las Heras, A. (1991): Navegar por la información. Madrid: FUNDESCO.

Romero, J., y Degrado, M. (2005): "Programas musicales en la televisión”, Comunicar, nº 25.

Sánchez Carballido, J. R. (2008): "Perspectivas de la información en Internet: ciberdemocracia, redes sociales, y web semántica”, Zer, nํ25, pp. 61-81.

Sánchez, A. (2011): Generación 2.0. Hábitos de usos de las redes sociales en los adolescentes de España y América Latina. Universidad Camilo José Cela.

Sedeño, A. (2005): "La necesidad de protección de la música como patrimonio cultural y artístico: piratería y antipiratería", Revista Latina de Comunicación Social, ํ 59.

Turtós, Jordi (2008) "Música y medios de comunicación en Cataluña”, en Aguilera, Miguel; Elies, Joan y Sedeño, Ana María. Comunicación y música. Barcelona: Editorial UOC, pp. 189-223. 
Yin, R. (1993): Applications of case study research, Newbury Park: Sage.

\section{Nota}

Investigación financiada por el Ministerio de Ciencia e Innovación de España. Proyecto I +D +i: Evolución de los cibermedios españoles en el marco de la convergencia. Distribución y tecnología. 


\section{Forma de citar este artículo en bibliografías}

BERNAL TRIVIÑO, A. I. (2012): "Jóvenes, música y pantallas. Evolución formal y de contenido de los 40.com y adaptación a los dispositivos móviles”, en Revista PANGEA, 3, páginas 64 a 90. Red Académica Iberoamericana de Comunicación. Recuperado el _ de de 2 de: http://www.revistapangea.org 